\title{
Antagonistic Coevolution and Sex
}

\author{
Curtis M. Lively
}

Published online: 7 January 2010

(C) Springer Science+Business Media, LLC 2009

\begin{abstract}
One of the leading hypotheses for the maintenance of sexual reproduction is the Red Queen hypothesis. The underlying premise of the Red Queen hypothesis is that parasites rapidly evolve to infect common host genotypes. This response by parasites could result in the long-term maintenance of genetic variation and may favor sexual reproduction over asexual reproduction. The underlying ideas present a wonderful microcosm for teaching evolution. Here I present the reasons for why sex is anomalous for evolutionary theory, the rationale underlying the Red Queen hypothesis, and some empirical studies of the Red Queen hypothesis using a freshwater snail. The empirical results are consistent with the Red Queen hypothesis. In addition, the distribution of sexual and asexual reproduction in the snail leads naturally to thinking about coevolution in a geographic mosaic of parasite-mediated natural selection.
\end{abstract}

Keywords Coevolution - Geographic mosaic theory ·

Host-parasite interactions · Red Queen hypothesis

\section{Introduction}

Why sex? In this paper, I will make the case that coevolution between hosts and parasites could lead to the long-term maintenance of genetic diversity in both players. Host-parasite coevolution might also lead to selection for sexual reproduction as a mechanism for producing genetically diverse offspring, which may be more successful at

C. M. Lively $(\bowtie)$

Department of Biology, Indiana University,

Bloomington, IN 47405, USA

e-mail: clively@indiana.edu escaping infection. First, I will try to convince you that sex is costly.

This is not as racy as it may sound. By "sex," evolutionary biologists mean genetic mixing between two individuals that results in offspring that are genetically different from each other and from their parents. Genetic mixing is the norm for most eukaryotes; but some plants and animals reproduce asexually through a process called parthenogenesis (virgin birth). Parthenogenesis, however, is not very common. Why not? There are good reasons to think that asexual reproduction should be the dominant form of reproduction on the planet, as I will now explain.

There is a large group-level advantage to asexual reproduction: every individual can produce offspring. Consider a population composed of two hundred individuals. Let's say 100 of them are asexual females, and the other 100 are sexual individuals, with 50 males and 50 females. Now let's assume that every female makes two offspring. How many sexual individuals and how many asexual individuals will be present in the next generation? Clearly, there would be more asexual individuals, but how many more, and why?

Hopefully, it is clear that an asexual population has a twofold reproductive advantage simply because every individual in the asexual population is capable of producing offspring. So why hasn't asexual reproduction become the most common method of propagation? Perhaps there are also some disadvantages of reproducing asexually that might counter the advantages. Keep in mind that the disadvantage would have to be very large (up to twofold) to overcome the fact that all asexual individuals are female and can give birth.

One major possibility should immediately come to mind. Asexual reproduction generates offspring that are all genetically identical (a clone). Genetic homogeneity could 
be a problem in a changing environment, as the asexual lineage might not be able to adapt to new conditions. This reasoning raises a new question: how long would it take an asexual clone beginning with a single individual to replace a sexual population of a certain size, say $1,000,000$ individuals? Any environmental change that selects against the clone would have to operate on this time scale or shorter, or the clone would win. The answer to this question is not intuitive, but I would encourage students to take a guess before continuing on. Keep in mind two things. First, the clone will double in number every generation until it becomes common. Second, the resources available for reproduction are limited, so the total population size cannot increase without bound.

By my calculations, the clone would replace the sexual population in less than 50 generations (Fig. 1). (Time to replacement is about seven times the log of the total population size). Think about that! A clone beginning with a single individual would drive a sexual population extinct in less than 50 generations, unless there are countervailing disadvantages to asexual reproduction that are manifested on the order of tens of generations. We thus seek an advantage to sexual reproduction that operates very rapidly, or we cannot explain why genetic exchange among individuals is so common. Clearly, we cannot appeal to long-term changes in climate, because they occur too slowly.

For these reasons, elucidating the possible advantages of sex is regarded as one of the major challenges of evolutionary biology, and the challenge has attracted the attention of numerous very gifted theoretical biologists. As it turns out, there are several viable possibilities, but I will try to explain one rather fascinating idea that involves the evolution of diseases. The idea is widely known as "the Red Queen Hypothesis."

First, let me ask another question. Answering the question for yourself may take you naturally to the gist of the idea; it may also cause you to ask other questions about modern agricultural practices.

Suppose you and your immediate family depend on a small garden for survival. In fact, your total food supply comes from one food crop in that garden. Do you want to plant a high-yielding monoculture, or would you prefer to plant a genetically diverse crop of the same species, which is expected to give a lower yield?

I have asked this question in many graduate and undergraduate courses in biology. Most students would favor the genetically diverse crop, which is akin to sexual reproduction. They favor diversity, even though I insist they will see a short-term reduction in yield. But the students also insist that they would prefer to take the short-term cost
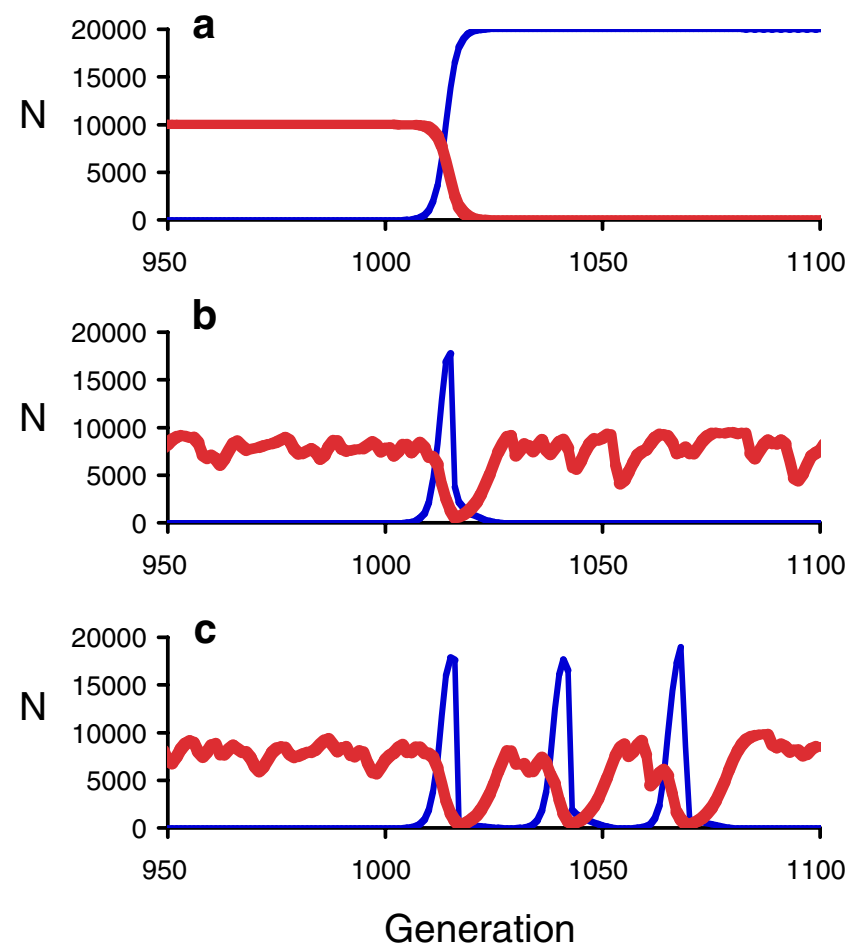

Fig. 1 The outcome of competition between a genetically diverse sexual population and a single asexual genotype. The results are based on computer simulations wherein a clone is introduced by mutation into a sexual population at generation 1000. a No parasite. In the absence of parasites, the clone rapidly eliminates the sexual population. The asexual clone also reaches a higher carrying capacity, because asexual females can replace themselves on fewer resources (see Lively 2009). b Virulent parasites present. Here, the clone increases after its introduction, driving the sexual population down; but parasites quickly evolve to infect the clone and drive it to extinction. The sexual population then increases to its previous density. c Virulent parasites present. Here, the clone invades the sexual population, and is subsequently driven down in frequency by parasites, but it does not go extinct. The clone then begins to oscillate in the population. Nonetheless, the parasites have prevented the clone from driving the sexual population to extinction. The simulation is described in Lively (2009). In b and $\mathbf{c}$, infected females produce one offspring, while uninfected females produce three offspring

to insure greater success in the long term. They are worried that pathogens would quickly evolve to infect the genotype represented in the high-yield clone, and that the monoculture's yield would crash. They have good reason to worry. This has happened many times in crop monocultures (Barrett 1988). (Note that I am not suggesting that natural selection has foresight; only that it can operate on the geometric mean, which depends on the product of relative fitness over time (Stearns 2000)).

If you selected the high-diversity crop, you will already see where I am going. Pathogens can adapt very quickly to infect common genotypes, and there may be some protection against infection in genetically diverse populations. These are the conceptual underpinnings of the Red Queen hypothesis. 
Imagine a sexual host population that is infected by parasites; assume that both the host and the parasite are genetically diverse for resistance and infectivity, respectively. Let's say that, for whatever reason, one of the host genotypes is very common in the population, while the rest are rare. In addition, Let's assume that each parasite genotype can only infect one host genotype. Clearly, the parasite genotype that can infect the most common host genotype will be the most successful at reproducing. This reproductive success for the parasite will then increase the likelihood for infection in the most common host genotype in the next generation. If the parasite reduces the fitness of infected hosts, then there will be selection against the most common host genotype, and that genotype will eventually be driven down in frequency. But now a new, previously rare, uninfected host genotype will become the most common genotype in the host population. And the cycle begins again; now any parasite strain that can infect this newly common host genotype will increase in frequency and drive that host genotype down, leading to the emergence of a new common host genotype in the population. So host and parasite genotypes would be expected to cycle over time (Fig. 2). It is as if both the host and the parasite are running as fast as they can, just to stay in the same place. Over long periods of evolutionary time, we would expect each of the genotypes to cycle between periods of being common and rare, and we would expect for there to be a high level of genetic diversity at genes encoding parasite resistance (Fig. 2). It is important to note that this cycling of genotypes is very different from an arms race, which might be expected between predator and prey. There is no net increase in resistance over time, but rather a cycling of previous used strategies.

This cycling of genotypes over time has reminded some evolutionary biologists of the verbal exchange between Alice and the Red Queen in Lewis Carroll's novel, Through the Looking Glass. I often read this passage to my classes, although, at first, they think I am crazy. Alice first passes through a mirror (the looking glass) into a very different world. In this world, the chess pieces are alive and walking around; and the flowers in the garden can talk. Actually, the flowers are rather rude to Alice. But the flowers are also the ones who tell Alice about someone who has funny petals, like hers, and who often passes by. Alice wants to meet this person and sets out after her. But she returns to the flowers without success. The rose (who is very opinionated) then gives Alice some key advice: go the opposite way. In doing so, Alice immediately comes face to face with the Red Queen. The Red Queen then leads Alice up a hill. She begins to run, and Alice runs after her. But it seems to Alice that they are not going anywhere, even though they are running as fast as they can. When they finally stop, the exhausted Alice remarks on this, and she adds that, in her country, one usually gets somewhere by running. The Red Queen replies with this very famous statement:

"Now, here, you see, it takes all the running you can do to keep in the same place."

Hence, the idea that host-parasite coevolution might drive oscillatory changes in genotype frequencies is called the Red Queen hypothesis (Bell 1982). The hypothesis may
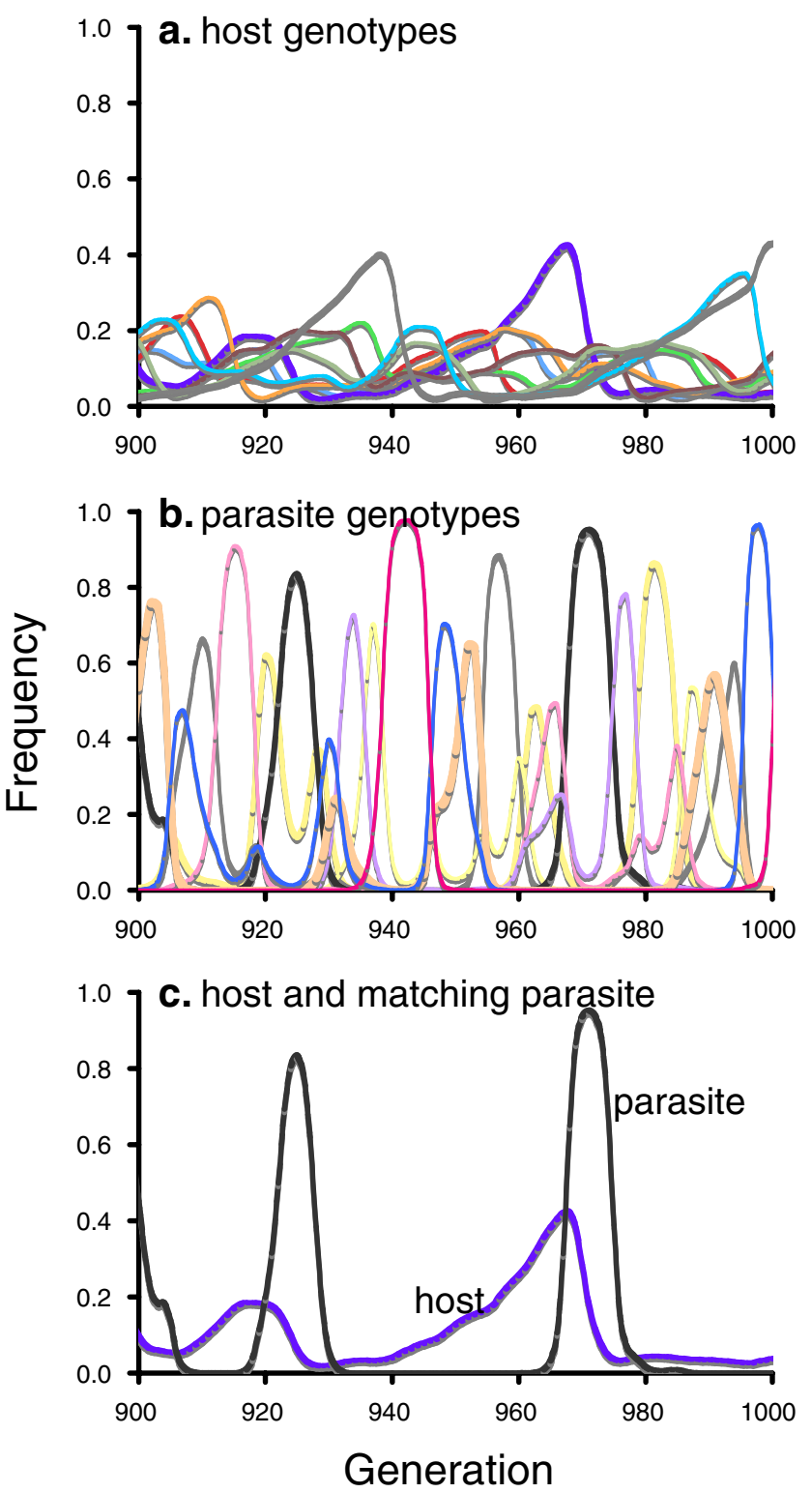

Fig. 2 Red Queen dynamics. a Oscillation of eight host genotypes in a sexual population of hosts. b Oscillation of eight parasite genotypes; infection requires that the parasite genotype "matches" the host genotype. c One host genotype, and its matching (infective) parasite genotype. Note that the parasite genotype is about 90 degrees out of phase with the host. Also note that the amplitude of the oscillation is much higher in the parasites population than in the host population. The results were generated using a computer simulation (Lively 2009) 
or may not be correct, but it has one of the coolest names in all of science.

The Red Queen hypothesis can then be stretched a bit to become a hypothesis for sexual reproduction. Suppose we introduce an asexual clone into a sexual host population. We would expect it to rapidly spread, due to the fact that all the clonal individuals give birth, but we might also expect parasites to evolve to disproportionately infect the clone after it becomes common. If infection is both common and virulent, the parasites might prevent the clone from replacing the sexual population (Fig. 1). We might expect further that the parasites would drive the clone down to a very low frequency. Thus, parasites could play some role in selecting for sexual over asexual reproduction.

The idea has a good deal of intuitive appeal, but such appeal is not enough. The question is: does the Red Queen hypothesis for sex hold up to empirical examination? It is, after all, only one of several viable hypotheses that are hotly debated among evolutionary biologists.

\section{Experimental Examination of the Red Queen}

How does one test the Red Queen hypothesis (RQH)? I would ask my class at this point to work in small groups to generate simple clean tests that would yield unambiguous data to decide the issue. The emphasis would be on designing tests that would falsify the RQH, rather than prove it. What data would falsify the RQH? I would have also warned them at the beginning of class that I was going to ask this question, and that they would have 15 minutes to answer it while working in groups. Given that they know the question, they tend to follow any leads given to them. I am very often surprised at how clever they are, and how quickly they can generate experiments. One thing for sure is that they will remember the Red Queen. Given the information at hand, here is a subset of possibilities for rejecting the RQH.

1. The hypothesis seems to rely very heavily on the assumption that genetic variation for resistance exists in the host population, and that each parasite genotype can only infect a small subset of the possible host genotypes. If all parasite genotypes (or random isolates for experimental purposes) can infect all host genotypes, the RQH is false.

2. The hypothesis seems to suggest that each parasite population is "chasing" host genotypes in the same location. Thus, even if the host genotypes are the same in different locations, there is no reason to think that all the populations would be at exactly the same point in the oscillatory dynamic. Hence, under the Red Queen, parasites would be expected to be better at infecting hosts collected from the same location than hosts collected from different locations.

3. If we had multiple host populations (also assumed above), we would expect to find more genetic diversity and more sexual reproduction in those locations where the risk of exposure to virulent parasites was greater. We would expect asexual reproduction to dominate wherever parasites were rare or absent. Otherwise, the $\mathrm{RQH}$ is false.

4. If we could find populations that have mixtures of sexual and common hosts, we would expect to find that common clones become disproportionately infected shortly after they become common, and then driven down in frequency. We realize that this would be a long-term experiment, but any alternative finding would falsify the RQH.

This list, of course, includes just a few of the possible examples. The main thing is to generate experiments that examine the necessary conditions for the hypothesis to remain viable. I find that I can easily lose my students if I start talking about facts before they have internalized the problem and made their own guesses about how to resolve it.

Some data now exist that examine these same expectations. From my point of view, the data cannot be construed to falsify the Red Queen, and so she remains viable. I will concentrate my exploration of the data on a freshwater New Zealand snail, but other systems have been used to make serious strides to understand the RQH, most notably water fleas (Daphnia; Ebert 2008).

The New Zealand snail (Potamopyrgus antipodarum) has some interesting characteristics that make it especially well suited to testing the Red Queen hypothesis. Most importantly, the species has both sexual and asexual forms; and it is widely distributed throughout New Zealand where it is easily the most common, easily observed invertebrate in the country. It exists in almost every freshwater habitat, including some of the most beautiful lakes on the planet. Some of the snail populations are entirely asexual, and some populations are mixtures of sexual and asexual snails. Importantly, the snails cannot switch between reproductive modes; they are either sexual (and diploid) or asexual (and triploid). In addition, they are infected by about a dozen different species of parasitic trematode worms.

These worms are very virulent (i.e., infection greatly reduces host fitness). I will concentrate here on one species of worm that is especially common and virulent. This worm has a complicated lifestyle, and some of the details are important to understanding the data. For example, the adult worms live in the intestines of ducks, but they do not seem to harm the ducks in any way. Inside the duck, the hermaphroditic adult worms produce cross-fertilized eggs, 
which exit the ducks in the feces. The eggs sink to the bottom of the lake, where by chance they become ingested by snails while foraging on algae. Following ingestion, the eggs hatch, and larval worms emerge. We don't know the details, but we assume the following. The larva burrows into the snail's gut. If the snail's immune system recognizes the worm, it kills it. If not, the larva begins to reproduce asexually. In 3 months, most of the snail's shell will be filled with hundreds of encysted parasite larvae; only the snail's head will remain intact. The snail will be completely sterilized, as the parasite will have completely consumed the reproductive organs of both male and female snails. However, the infected snails do not die. The larval cysts "hatch" only after the infected snails are eaten by ducks (Remember this key point). The larvae mature into hermaphroditic adults, which make the next generation of eggs. The two-host life cycle is complete. In some places, up to 80 percent of the snails are sterilized by this parasite. In other places, the parasite is completely absent. No ducks, no parasite.

Now we can make some predictions related to the RQH that are specific to this host-parasite interaction. One is that asexual reproduction will replace sexual reproduction in the absence of parasites. If so, we would expect a pattern among natural populations showing that asexual reproduction is more common when parasites are rare or absent. I tested this prediction by examining large samples of snails from 62 different lakes across New Zealand (Lively 1992). For each sample, I determined the frequency of males and females and the frequency of infected males and females. The results were consistent with expectation under the $\mathrm{RQH}$. There were more all-female, asexual populations where infection was low; and there were more sexual populations where infection was common. This result is not proof of the Red Queen, but the data could easily have falsified the hypothesis. Moreover, we recently found that the same pattern exists in stream populations of the snail (King and Lively 2009). So among both lakes and streams, there is geographic variation in parasite prevalence, and asexual reproduction is more common where the parasites are rare.

A sensible alternative hypothesis immediately emerges. Perhaps there are fewer infected individuals in asexual snail populations, because asexual snails are more resistant to infection than sexual snails. However, field data suggest that males are not more infected than females (Lively 1987), and that, on average, asexual clones are not more resistant to infection than sexual females (Jokela et al. 1997).

The overall pattern observed among lake and stream populations of this snail is consistent with John Thompson's ideas regarding a geographic mosaic of coevolution (Thompson 1994, 1999). Specifically, the results are consistent with the idea that there are hot spots and cold spots across space; in hot spots, reciprocal selection and reciprocal adaptation is intense, while in cold spots, the interaction is less intense and perhaps swamped out by other factors. In the snail, it would seem reasonable to suggest that sexual females are favored in the coevolutionary hot spots where parasites are common, and that asexual females are favored in the coevolutionary cold spots where parasites are rare or absent. We recently tested these ideas more directly, and over much smaller spatial scales.

We have known since 1994 that there is a depth-related cline in the relative frequency of sexual females in Lake Alexandrina on the South Island of New Zealand. Lake Alexandrina is a wildlife preserve very near to the backbone of the Southern Alps, which runs through most of the South Island. The lake is on the east side (the dry side) of the Alps, not far from the spectacular Mt. Aoraki (or Mt. Cook). The cline is as follows: as one goes from the shallow-water margins of the lake to the deep-water (5-6 meters) vegetation, the frequency of sexual individuals decreases from very high (about 90 percent) to very low (less than 10 percent). My collaborator, Jukka Jokela, discovered this cline on his first trip to New Zealand (Jokela and Lively 1995a, 1995b). This is an amazing finding, as the deep and shallow subpopulations are not separated by barriers. In later experiments, we found that the parasites from the same lake were much better at infecting snails from the shallow water than snails from the deep water, independent of the depth where the parasites were collected (Lively and Jokela 1996). Finally, we spent a lot of time watching the ducks on the lake, and we noted their foraging location in relation to small buoys that we set to mark different depths. We found that the dabbling ducks were only foraging in the shallow water $(<2$ meters), and the diving ducks were mainly foraging in water of 1-3 meters (Jokela and Lively, unpublished observations). In other words, none of the ducks were foraging in the deepwater vegetation at 4-6 meters.

We constructed a hypothesis. To fully understand the hypothesis, it is helpful to emphasize that the RQH is not just about infection by parasites but about coevolution between host and parasites. The idea is that coevolution by parasites is restricted to the shallow-water regions of the lake $(<3$ meters), because this is where the ducks are foraging, and ducks are the final host for the parasite. Any infections in the deep water would not be "recycled" by the ducks, and hence, they (the parasites) are out of the coevolutionary loop (living in a deep blue coevolutionary cold spot). So the hypothesis is that parasites will be more infective to snails collected from the shallow water than to snails collected from the deep water (Fig. 3).

However, there is also a very sensible alternative hypothesis that any study would have to consider. The 


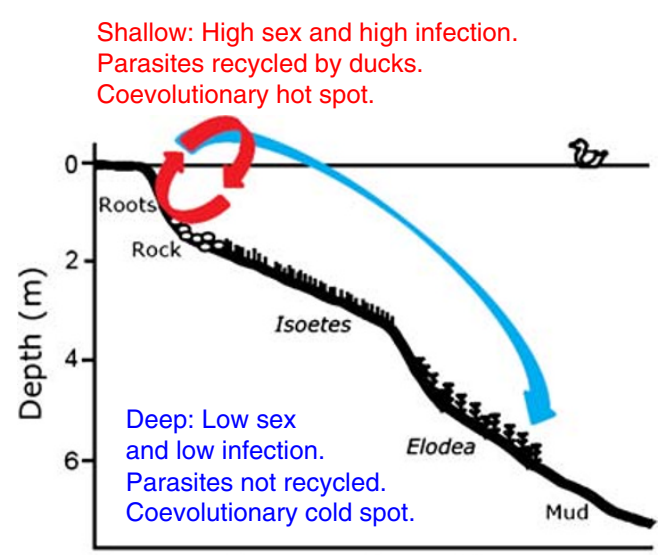

Distance From Shore

Fig. 3 Cross section of Lake Alexandrina, showing depth-specific habitat zones and hypothesized gene flow (redrawn from King et al. (2009)). Data from this lake show that sexual snails are more common in the shallow-water habitats, and asexual snails are more common in the deep-water habitats. Infection by trematode worms is also more common in the shallow water. Because parasite larvae must be ingested by ducks and because ducks forage mainly in the shallow water $(<3$ meters), we hypothesized that parasite genes would more likely be recycled in the shallow water. Infections in the deep water would be out of the coevolutionary loop. Hence, we predicted that local parasites would be more infective to shallow-water hosts than deep-water hosts

alternative is that shallow-water snails are more infected by parasites simply because they are more susceptible to infection in general. Hence, we wanted to design an experiment that would decide between the two alternative hypotheses: (1) the geographic mosaic hypothesis concerning coevolutionary hot spots (shallow) and cold spots (deep), and (2) the inherent susceptibility hypothesis, which posits that shallow-water snails are simply more susceptible to infection than deep-water snails.

How to contrast these two alternatives? (I would ask my class for suggestions.) Here is how we did it (King et al. 2009). We reasoned that, if snails collected from the shallow water were inherently more susceptible to infection, then they would be more susceptible to infection, regardless of the source of the parasites. So if we collected parasites' eggs from another lake, then they should infect the shallow-water snails significantly more often than the deep-water snails. On the other hand, if shallow-water snails were more susceptible as a result of being in a coevolutionary hot spot, then the shallow-water snails should only be more susceptible to parasites collected from the same lake, but not more susceptible to parasites collected from a different lake.

To contrast the hypotheses, we collected snails from the shallow and deep regions of two different lakes (Lake Alexandrina and Lake Kanieri, which are on the opposite sides of the Southern Alps). We also collected the droppings from ducks at both lakes, assuming that these droppings would contain the eggs from the local parasites. After rinsing the duck feces for several days in fresh water, we randomly distributed the slurry to each of the different snail collections (Alexandrina shallow, Alexandrina deep, Kanieri shallow, and Kanieri deep).

We found that the shallow-water snails from both lakes were more infected than the deep-water snails, but only by the local source of parasites. For example, shallow-water snails from Lake Alexandrina were more infected than deep-water snails, but only by the parasites from Lake Alexandrina (Fig. 4). The parasites from Lake Kanieri showed no difference in infectivity to shallow- and deepwater snails from Lake Alexandrina. Hence, the "inherent susceptibility" hypothesis is rejected. The results are in striking accord with the coevolutionary hot spot hypothesis. It is therefore reasonable to suggest that sexual reproduction in the shallow-water margins of Lake Alexandrina may be a consequence of coevolutionary interactions with virulent parasites (King et al. 2009).

We now also have another test of the Red Queen hypothesis (Jokela et al. 2009). Remember that the hypothesis predicts that, if a clone spreads into a sexual population, parasites should quickly evolve to dispropor-

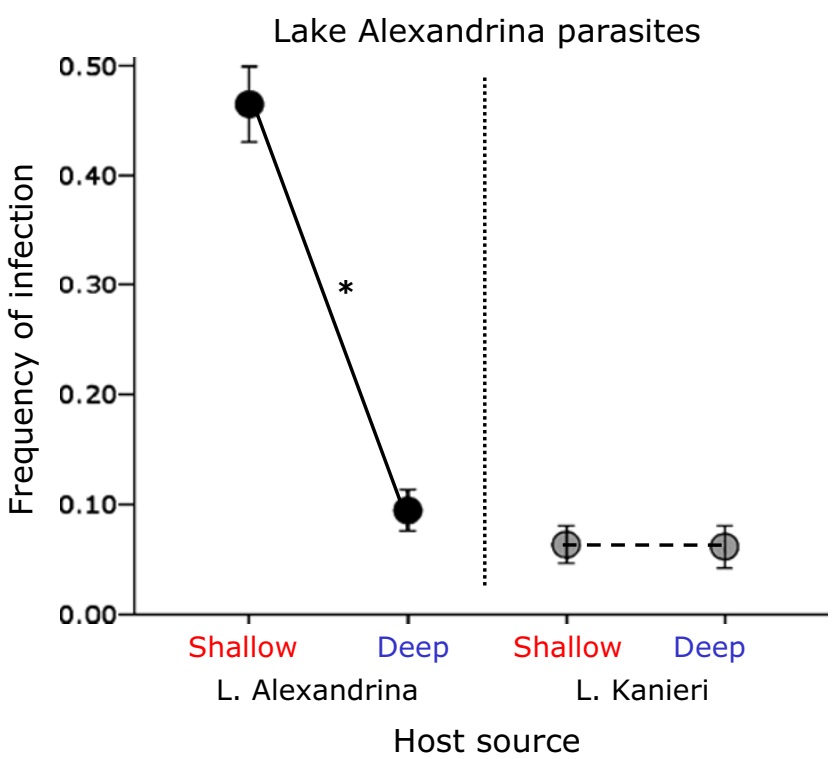

Fig. 4 Results from laboratory cross-inoculation experiment. The graph gives the mean infection frequencies for snails exposed to parasites' eggs collected at Lake Alexandrina. The vertical bars give one standard error of the means. The asterisk indicates a statistically significant difference. Note that the parasites collected from Lake Alexandrina were significantly more infective to snails collected from the shallow-water margins of the same lake than to snails collected from the deep water. Also note that the Lake Alexandrina parasites were no more infective to shallow-water snails collected from Lake Kanieri than to deep-water snails from Lake Kanieri. These results suggest that the shallow-water margin of Lake Alexandrina is a coevolutionary hot spot, while the deep habitat is a coevolutionary cold spot 
tionately infect the clone, and drive it down in relative frequency. We have been observing the clonal dynamics in the shallow-water margins of Lake Alexandrina for 15 years. In 1994, there were several common clonal genotypes that were coexisting with the sexual population of snails. These common clones were also less infected than expected by chance, given the infection frequencies in the lake. We then sampled these populations again 7-10 years later to determine the fate of these clones. Amazingly, we found that the common clones from 1994 had been dramatically reduced in frequency, and that they were also more susceptible to infection. A previously rare clone was dominant in the population, and it was almost free of infection. This is exactly what is expected under the Red Queen hypothesis. We also found this exact same result in a laboratory study (Koskella and Lively 2007; Koskella and Lively 2009), further convincing us that parasites, rather than correlated environmental factors, are driving the observed changes.

\section{Conclusions}

Our results suggest that hosts and parasites are coevolving in natural populations of snails and trematode worms. In addition, we find hot spots (shallow) and cold spots (deep) within the same lake, and we find that sexual reproduction in the snail is associated with the coevolutionary hot spots. The results are consistent with expectation under the Red Queen hypothesis. It would thus seem that the Red Queen is alive and well, living in a geographic mosaic.

Acknowledgment I thank Britt Koskella, Kayla King, Maurine Neiman, John Thompson, Rodrigo Medel, and an anonymous reviewer for comments on the manuscript. I also thank the members of team Potamo, especially Jukka Jokela, Mark Dybdahl, Maurine Neiman, Erik Osnas, Britt Koskella, and Kayla King. Our work in New Zealand has been supported by the US National Science Foundation.

\section{References}

Barrett JA. Frequency-dependent selection in plant-fungal interactions. Proc R Soc Lond, B Biol Sci. 1988;319:473-82.

Bell G. The masterpiece of nature: the evolution and genetics of sexuality. Berkeley: University of California Press; 1982.

Ebert D. Host-parasite coevolution: Insights from the Daphniaparasite model system. Curr Opin Microbiol. 2008;11:290-301.

Jokela J, Lively CM. Parasites, sex, and early reproduction in a mixed population of freshwater snails. Evolution. 1995a;49:1268-71.

Jokela J, Lively CM. Spatial variation for infection by digenetic trematodes in a population of freshwater snails (Potamopyrgus antipodarum). Oecologia. 1995b;103:509-17.

Jokela J, Lively CM, Fox JA, Dybdahl MF. Flat reaction norms and "frozen" phenotypic variation in clonal snails (Potamopyrgus antipodarum). Evolution. 1997;51:1120-29.

Jokela J, Dybdahl M, Lively CM. The maintenance of sex, clonal dynamics, and host-parasite coevolution in a mixed population of sexual and asexual snails. Am Nat. 2009;174:S43-53.

King KC, Lively CM. Geographic variation in sterilizing parasite species and the Red Queen. Oikos. 2009;118:1416-20.

King KC, Delph LF, Jokela J, Lively CM. The geographic mosaic of sex and the Red Queen. Curr Biol. 2009;19:1438-41.

Koskella B, Lively CM. Advice of the rose: experimental coevolution of a trematode parasite and its snail host. Evolution. 2007;61:152-59.

Koskella B, Lively CM. Evidence for negative frequency-dependent selection during experimental coevolution of a freshwater snail and a sterilizing tremotode. Evolution. 2009;63:2213-21.

Lively CM. Evidence from a New Zealand snail for the maintenance of sex by parasitism. Nature. 1987;328:519-21.

Lively CM. Parthenogenesis in a freshwater snail: reproductive assurance versus parasitic release. Evolution. 1992;46:907-13.

Lively CM. The maintenance of sex: host-parasite coevolution with density-dependent virulence. J Evol Biol. 2009;22:2086-93.

Lively CM, Jokela J. Clinal variation for local adaptation in a hostparasite interaction. Proc R Soc Lond, B Biol Sci. 1996;263:89197.

Stearns SC. Daniel Bernoulli (1738): evolution and economics under risk. J Biosci. 2000;25:221-28.

Thompson JN. The coevolutionary process. Chicago: University of Chicago Press; 1994.

Thompson JN. Specific hypotheses on the geographic mosaic of coevolution. Am Nat. 1999;153:S1-S14. 